\title{
DRR and Portal Image Registration for Automatic Patient Positioning in Radiotherapy Treatment
}

\author{
Ma Consuelo Bastida-Jumilla • Jorge Larrey-Ruiz • \\ Rafael Verdú-Monedero • Juan Morales-Sánchez • \\ José-Luis Sancho-Gómez
}

Published online: 1 April 2011

(C) Society for Imaging Informatics in Medicine 2011

\begin{abstract}
Image processing turns out to be essential in the planning and verification of radiotherapy treatments. Before applying a radiotherapy treatment, a dosimetry planning must be performed. Usually, the planning is done by means of an X-ray volumetric analysis using computerized tomography, where the area to be radiated is marked out. During the treatment phase, it is necessary to place the patient under the particle accelerator exactly as considered in the dosimetry stage. Coarse alignment is achieved using fiduciary markers placed over the patient's skin as external references. Later, fine alignment is provided by comparing a digitally reconstructed radiography (DRR) from the planning stage and a portal image captured by the accelerator in the treatment stage. The preprocessing of DRR and portal images, as well as the minimization of the non-shared information between both kinds of images, is mandatory for the correct operation of the image registration algorithm. With this purpose, mathematical morphology and image processing techniques have been used. The
\end{abstract}

\footnotetext{
M. C. Bastida-Jumilla $(\bowtie) \cdot J$. Larrey-Ruiz •

R. Verdú-Monedero $\cdot$ J. Morales-Sánchez $\cdot$ J.-L. Sancho-Gómez

Tecnologías de la Información y las Comunicaciones Department,

Universidad Politécnica de Cartagena,

Campus Muralla del Mar Antiguo Cuartel de Antigones,

s/n 30202 Cartagena, Spain

e-mail: consuelo.bastida@upct.es

J. Larrey-Ruiz

e-mail: jorge.larrey@upct.es

R. Verdú-Monedero

e-mail: rafael.verdu@upct.es

J. Morales-Sánchez

e-mail: juan.morales@upct.es

J.-L. Sancho-Gómez

e-mail: josel.sancho@upct.es
}

present work describes a fully automatic method to calculate more accurately the necessary displacement of the couch to place the patient exactly at the planned position. The proposed method to achieve the correct positioning of the patient is based on advanced image registration techniques. Preliminary results show a perfect match with the displacement estimated by the physician.

Keywords Radiotherapy · Image registration · Image feature enhancement $\cdot$ Biomedical image analysis

\section{Motivation and Background}

Radiotherapy treatments (RT) are intended to irradiate a target volume while preserving nearby healthy tissues. Thus, it is crucial to guarantee that the prescribed dose is correctly supplied by checking dosimetric and geometric data before and during the treatment. Thereby, possible movements of the patient [1], positioning errors, human error due to the instrumentation handling [2], etc. must be solved before irradiating the patient.

The usual procedure in radiotherapy treatments comprises different stages. Firstly, a volumetric study based on computerized tomographies (CT) of the tumour and neighbouring organs under risk is performed. At this stage, at least three fiduciary markers are drawn on the patient's skin as external references.

Using data from the $\mathrm{CT}$, a planning system delimits the target volume. The planning system used in this work was the Pinnacle Radiation Therapy Planning Computer System from Adac Laboratories. This software is employed by the Hospital Universitario Virgen de la Arrrixaca (Murcia, Spain), which has provided all the data for this study. 
The treatment is later planned obtaining several digitally reconstructed radiography (DRR) images, whose energy ranges from 80 to $120 \mathrm{KeV}$. The calculated DRRs will serve as internal reference for the patient positioning, calculating as many DRRs as radiation incidence directions have been scheduled for the treatment. Finally, geometric and dosimetric data are sent to the linear particle accelerator in DICOM-RT format (see Fig. 1a-b).

After the planning stage, the patient must be correctly placed under the linear accelerator, so that the administered radiotheraphy treatment matches the previously planned treatment. The couch is then positioned by using the fiduciary markers on the patient's skin until there is a perfect match between the field over the patient and the planned one. Afterwards a purely visual comparison is performed between a DRR from the planning step and a portal image obtained in the accelerator by the iView system of Elekta (see Fig. 1c-d). Unlike conventional radiographies, the portal image appears very diffuse owing to the great energetic dispersion related to the high photonic energy levels - in the range of 6 to $25 \mathrm{MeV}$. The final goal consists of placing manually the patient under the accelerator, making the differences between the two previous images (DRR and portal) minimal.

It is noteworthy that at least one portal image (to a maximum of three according to the Hospital's protocol) is taken for each radiation angle and that all the procedure is repeated every new session of radiotherapy. This procedure is slow, subjective, imprecise, prone to errors and, thereby, potentially harmful for the patient.

To improve this method, the authors propose an image processing system that automatically compares DRRs and portal images using image registration techniques. The parameters defining the registration automatically lead to the proper correction of the patient positioning. It would also be desirable that this solution was automatic, fast (computationally efficient-since it is a real-time process, with the patient under the linear accelerator), precise (with an acceptable error under $1 \mathrm{~mm}$ ) and independent of the anatomical areas which are about to be radiated [3].

From its very beginning, it is known the ill-posedness of the image registration problem. This poor conditioning is caused, to a greater or lesser extent, by the presence of nonshared information between the images [4]. Although geometric and dosimetric data are sent to the accelerator in DICOM format, the provided tools at Hospital Universitario Virgen de la Arrixaca are proprietary system, which hinders the registration task. Besides, it is quite common not to have access to bare not treated images (i.e. raw data) in clinical practice. This will be our scenario, on the one hand, the DRR images provided by the planning system show two superimposed ortogonal axes together
Fig. 1 Necessary elements for the planning and treatment stages: a planning software Pinnacle; b DRR; c portal image; and $\mathbf{d}$ software used for visual validation of the positioning
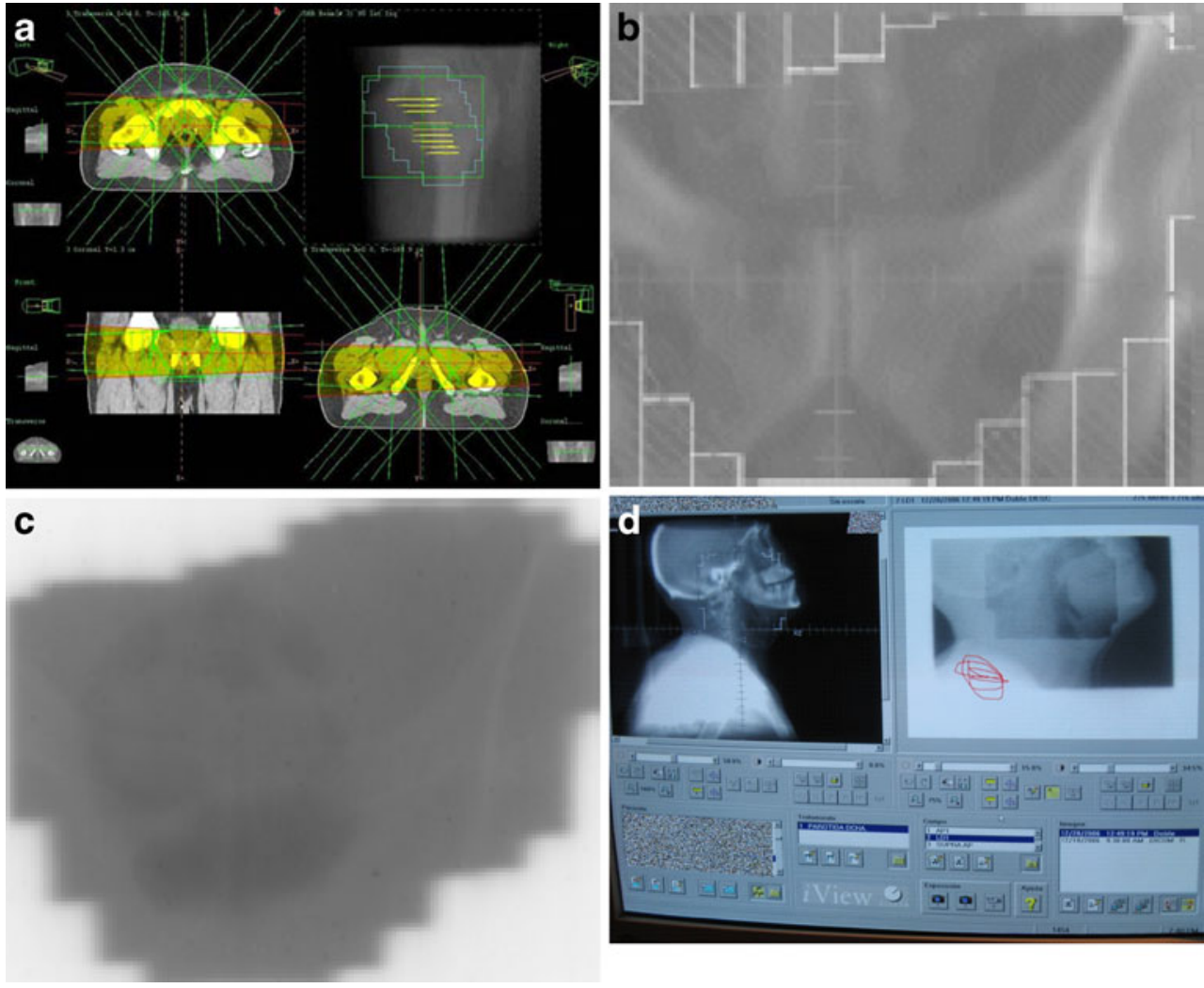
with their corresponding markers, the jaw (or multileaf collimator which forms the radiation beam in the accelerator) and a great amount of auxiliary text with information related to the treatment. On the other hand, double exposure portal images show the "accelerator print" or "collimator print", which can be noticed as a change in the mean intensity level in the area inside the radiation field (area influenced by radiation,see Fig. 1c) with respect to the external area (where no radiation should be absorbed, except for the dispersion phenomenon). From previous reasoning, the need of preprocessing both images can be easily inferred. By preprocessing DRR and portal images before the registration, we want to minimize the non-shared data between them, such as the axes, the text or the collimator print.

Several attempts to register DRRs and portal images appear in the literature, but they do not share the same initial conditions with our problem. Matsopoulos et al. [5] use portal images in which only the radiation field appears. Clippe et al. [6] and Kim et al. [7] test the registration method on phantoms, but introducing markers inside the phantom to help the registration. Künzler's method [8] requires only a simple equalization of the portal image to register it, while Khamene et al. [9] work with portal images without accelerator print.

In the following sections, the proposed methodology is detailed: DRRs cleaning, equalization of portal images and registration procedure. Some application examples are also included. Finally, preliminary results obtained in the real scenario hereby presented are discussed.

\section{Proposed Methodology}

\section{DRR Cleaning}

This section describes an automatic process to detect axes, text and the collimator print in DRR images. This process is based on morphological operators, mainly opening and reconstruction. Later, only the pixels of the image covered by these elements are restored.

Mathematical morphology operators are non-linear tools which can extract image components taking into account the shape of the objects $[10,11]$. In our scenario, DRR images contain linear structures, with horizontal or vertical direction, that have to be detected and removed. In the case of DRR images with rotated axes, a filtering of the oriented structures with orientated space-variant structuring elements could be done.

The supplied images by the software Pinnacle are $770 \times$ 770 pixels (see Fig. 2a). Firstly, characters X, Y, 1 and 2 are located by using correlation, and they are later stored in a binary mask (as shown in Fig. 2b). An opening with a horizontal structuring element of length 615 pixels provides the grey level of the axes. Horizontal and vertical axes are extracted (see Fig. 2c) by means of two openings with structuring elements of sizes $1 \times 399$ and $399 \times 1$, respectively.

The box delimiting the area to radiate (collimator) is obtained by subtracting the axes from the binary mask containing the pixels in the DRR with the same intensity as the axes (see Fig. 2d). After that, the maximum of two new openings with structuring elements of size $1 \times 51$ and $51 \times 1$ (see Fig. 2e) is calculated.

The axis markers are extracted by using an opening of the image after subtracting the axes and the box from the previous mask. Once all the undesirable elements in the DRR have been located (Fig. 2f), only the pixels covered by these elements are restored. Since all elements are one pixel wide, the gray value of the pixel is obtained via a linear interpolation, i.e. by convolving the image with the filters $h_{\mathrm{h}}=0.5\left[\begin{array}{lll}1 & 0 & 1\end{array}\right]$ and $h_{\mathrm{v}}=0.5\left[\begin{array}{lll}1 & 0 & 1\end{array}\right]^{T}$.

The next step is to remove text from the DRR with a morphological reconstruction, where the mask is the image without axes, and the marker is the mask eroded with a structuring element $7 \times 3$. The application of a threshold to the difference between the original and the reconstructed DRR provides a binary mask with the text.

Finally, a space-variant filter is applied to restore the gray level of the text pixels in the image (see Fig. $2 \mathrm{~g}$ ). This filtering averages intensities using a window $9 \times 9$ pixels, but taking into account only non-text pixels. The result after all these operations is shown in Fig. $2 \mathrm{~h}$.

\section{Portal Images Equalization}

The automatic procedure used to eliminate the accelerator print in the portal image (see Fig. 3a) involves two phases: location of the accelerator print over the portal image and processing of that area to correct the intensity change without affecting the rest of the image. Previous efforts in the field of radiation edge detection have been made [12, 13]. However, these methods were not applicable to our problem, mainly because the provided portal images were very diffuse; they even present a width in the transition of the radiation field over 12 pixels.

\section{Accelerator Print Location}

The available accelerator print data are related to the shape of the radiation beam, which is stored in a plain text file with prs extension obtained from the particle accelerator. This file contains the displacements of every leaf which the jaw is made of. From this file, the shape of the accelerator print can be inferred and later translated into a binary mask (see Fig. 3c). To establish not only its shape but also its 
Fig. 2 Automatic DRR cleaning: a original image; $\mathbf{b}-\mathbf{g}$

extracted masks from original

image as described in "DRR

Cleaning"; (h) processed DRR
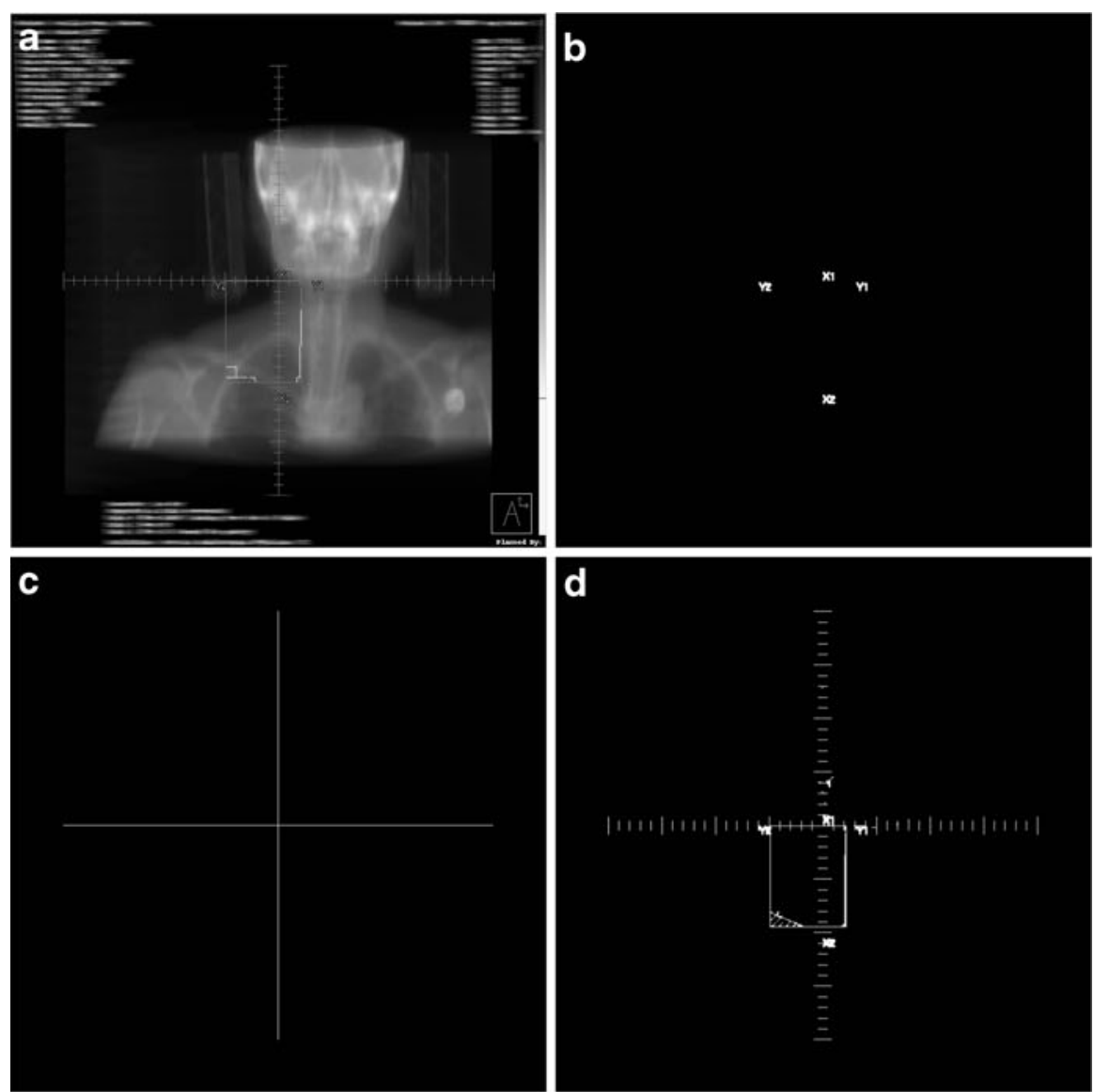

d

e

f
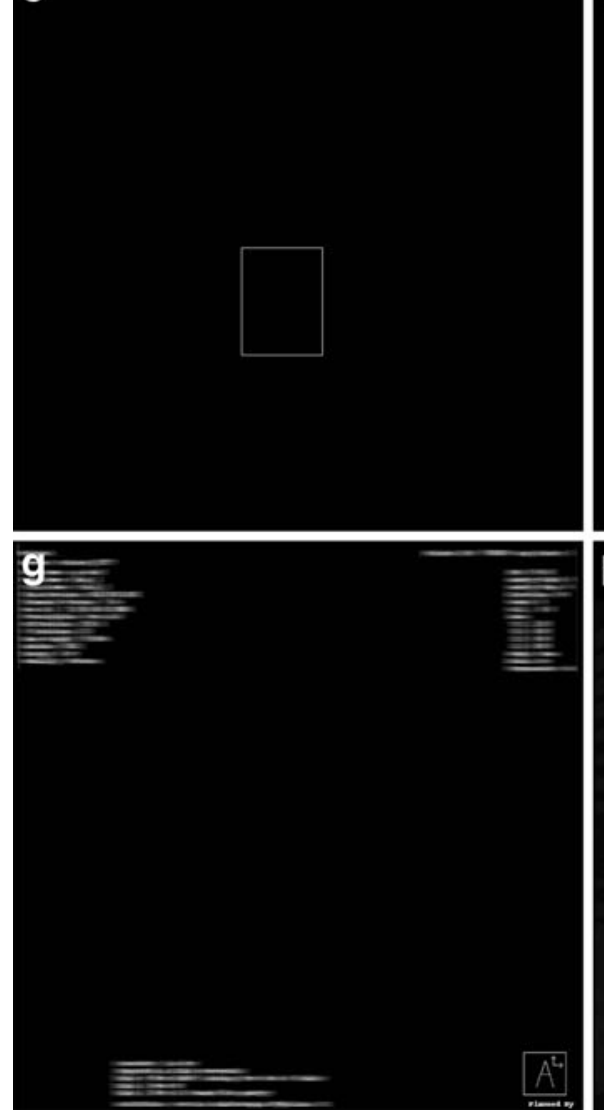

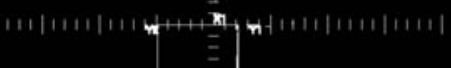

$\bar{x}$

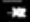

$=$
$=$

h

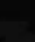


Fig. 3 Portal image cleaning, first phase: a original image; b portal image edges; c mask of the accelerator print; d edges from the accelerator print mask
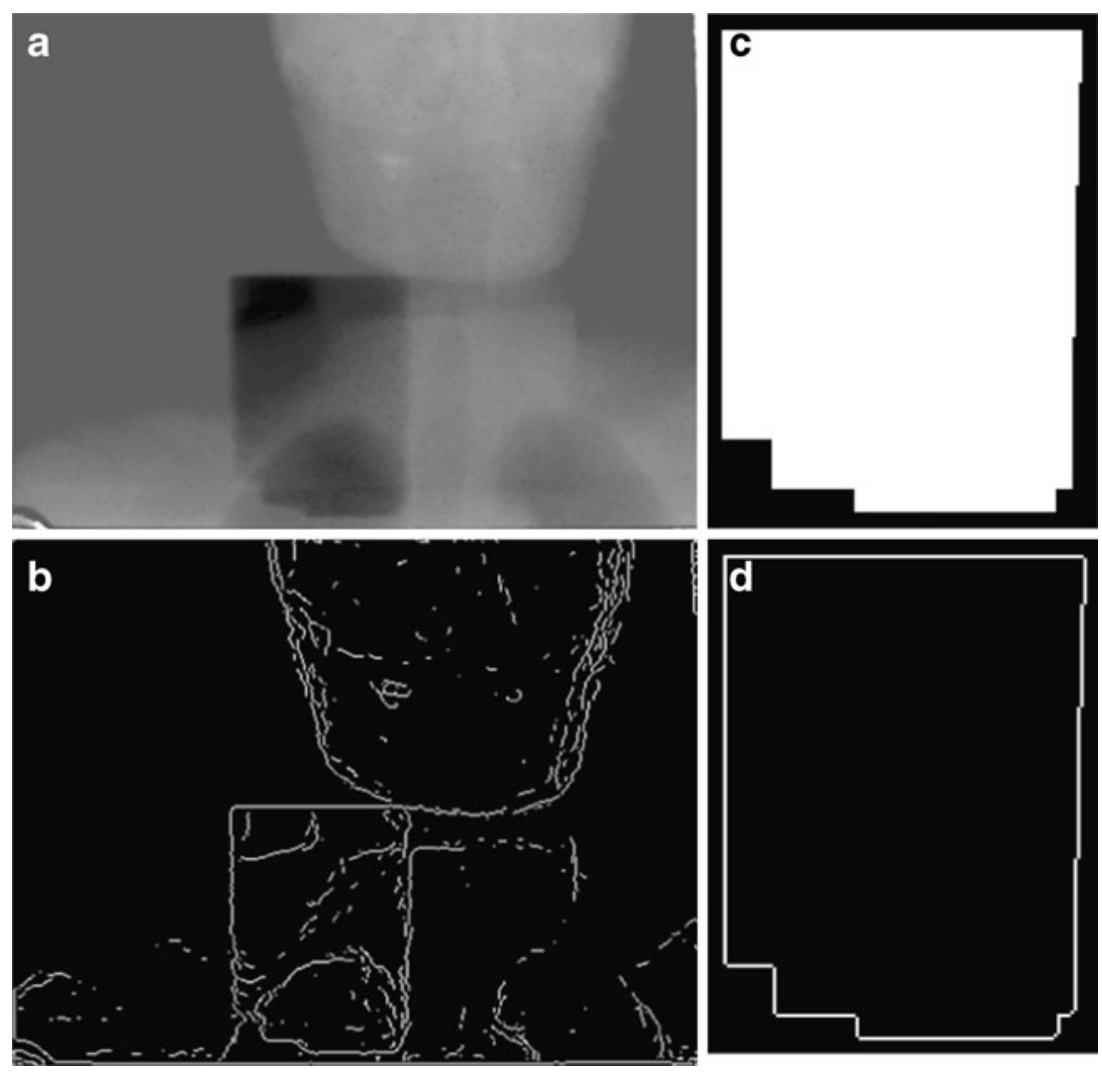

size, a correlation operation between the edges of the portal image and the mask edges is performed.

Although the correlation step gave us an initial approximation of the scale of the image, empirical practice showed that every image presents small variations. Besides, the scale differences were not comparable for horizontal and vertical directions. The scale variations, which affect the portal image, were probably caused by the position of the couch, the angle and distance from the image acquisition system or both of them.

Since one of the main well-known properties of a median filtering is the ability to reduce impulsive noise in the image while preserving borders, a $5 \times 5$ pixel wide median filter is applied over the portal image to find the optimal scale for each direction. After filtering, the adjustment of the scale is made via a fine scan with a small range of $\pm 2.5 \%$ with respect to the size of the image in both directions. A correlation operation between medianfiltered portal image and an edge-dilated version of the binary mask is performed for both directions, taking the maximum of correlation results from all the scales as the scale correction value. The edge detector used to extract the edges from the binary mask is Sobel's.

Once the scale correction has been calculated, the mask is resized using linear interpolation, making the accelerator print in both images, portal and mask, of the same size. Finally, another median filtering and correlation process are carried out to locate the position of the accelerator print on the portal image (see Fig. 3b, d).

\section{Accelerator Print Deletion}

For the second phase, which attempts to delete the accelerator print, the intensity of the portal image must be adjusted. First of all, a homomorphic filtering is applied so as to level the illumination in the portal image. A first-order high-pass Butterworth filter has been chosen. The applied transfer function is

$$
H(u, v)=\frac{1}{1+\left[\frac{1}{D_{0}} \sqrt{(u-M / 2)^{2}+(v-N / 2)^{2}}\right]^{2}}
$$

where $D_{0}=100$ is the cutoff frequency, $M$ and $N$ are the number of rows and columns of the original image, respectively, and $(u, v)$ represent the coordinates in the Fourier domain. The homomorphic filtering output for image in Fig. 5a can be seen in Fig. 5b, where the illumination normalization is noticeable.

To remove the accelerator print in the portal image, a new print mask with three regions is considered (see Fig. 4a): the internal region, formed by the pixels modified at this stage or the pixels inside the accelerator print area, the external region, formed by all those pixels outside the 

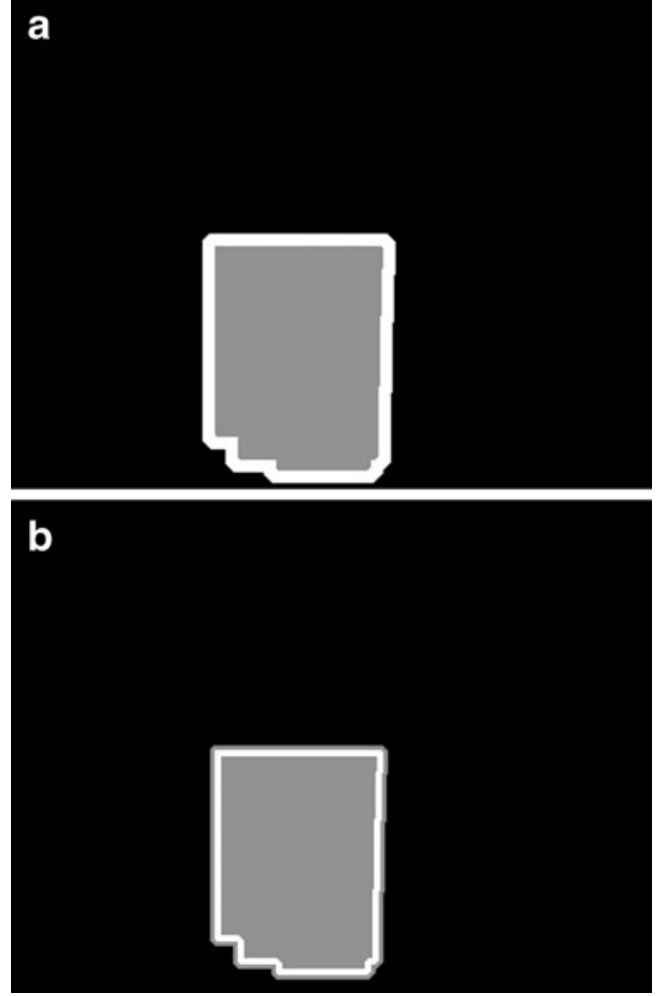

Fig. 4 Interest regions in portal image: a internal region (grey) and border region (white) considered for processing; $\mathbf{b}$ external (black and external grey edge), internal (inner grey and white areas) and transition (outer grey and white edges) regions internal region, and the border region (of the internal area), composed of a 9 pixels wide contour adjacent to the internal region border. To delimit these regions morphological processing with a disc-shaped structuring element has been used.

The processing of the pixels in the inner region follows two principles: global and individual. For the individual processing, the mean at the border region $m$ (see Fig. $4 \mathrm{a}$ ) is computed and individually compared with the mean of the inner region pixels. If the intensity of the pixel is $\mathrm{p}^{\prime}(\mathrm{i})<\mathrm{m}+20$, its value is updated to $\mathrm{p}^{\prime}(\mathrm{i})$ as follows

$p^{\prime}(i)=p(i)+10+(m-p(i)) / 2$

and if $p^{\prime}(i) \geq m+20$, then the pixel value will not be modified.

In the global processing scheme, the mean of the internal region after individual processing is calculated. The intensity value of the internal region pixels will be increased or decreased by a fixed value until the intensity means of the inner border regions are equal. The result of this processing can be seen in Fig. 5c. Although the intensity mean inside the accelerator print area is now more similar to the one outside the print, a sharp edge can be still noticed at the border.

At this point, another zone is defined, the transition region, which is a 9 pixels wide area covering points of both the internal and external regions (see Fig. 4b). To avoid the sharp edge in Fig. 5c, transition region pixels are linearly interpolated. The result of the interpolation can be
Fig. 5 Portal image cleaning, second phase: a portal image with the accelerator print over it; b result after homomorphic filtering; c result after processing the internal and border regions; d result after smoothing the border
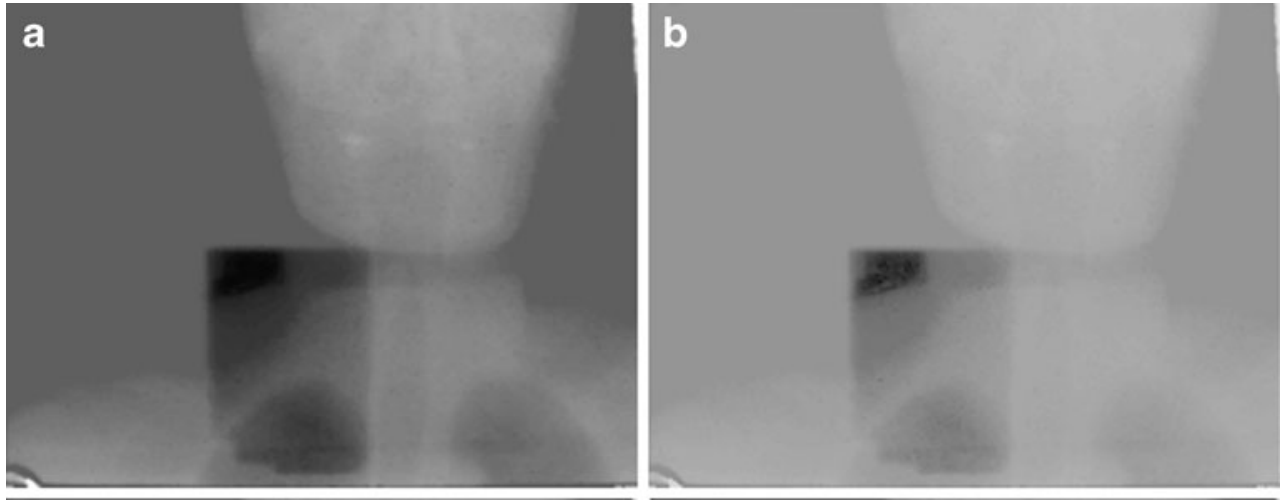

c

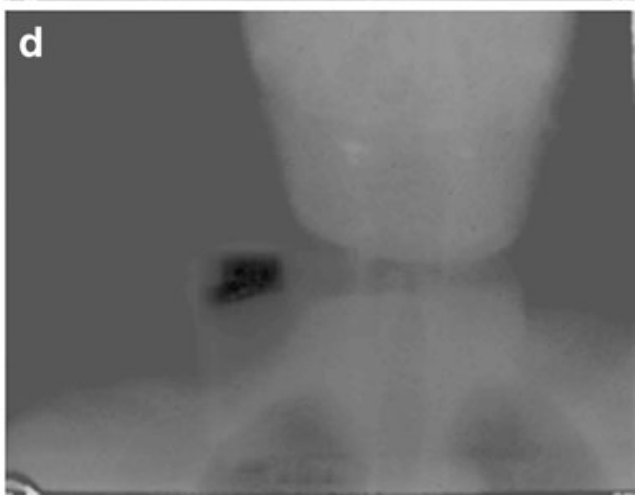


seen in Fig. 5d, where the sharp edge has been smoothed making the accelerator print much less noticeable.

\section{Image Registration Procedure}

Geometrically, image registration involves "aligning" one of the images, known as template image (the portal image in our application), and another image, the so-called reference image (the DRR in the positioning problem). After the registration process, a vector field representing the displacement that could transform the template image into the reference image is obtained. In a variational framework, the registration problem can be formulated as follows [4]: given two images, a reference $(R)$ and a template $(T)$, and a positive regularization parameter $(\alpha)$, we wish to find the displacement field that minimizes a determined cost function, i.e.,

$u(x)=\min _{u}\{D[R, T ; u]+\alpha S[u]\}$

being $(D)$ the energy term which measures the disparity between the images and $(\alpha)$ the regularization term, which penalizes the irregularities in the estimated displacement field. The parameter $\alpha$, is a scalar that weighs the influence of the second term of the functional versus the first one.

The whole registration procedure is shown in the flow diagram in Fig. 6, where the optimizer block implements the expression in (Eq. 3), driving the transformation to converge to its optimal. Other necessary elements in the registration process are the calculated transformation (which is computed in each iteration), the metric method imposed, which indicates how close to the optimal solution we are, and the interpolator, used to apply the considered transformation to the reference image.

Since the DRR and the portal image are obtained with different radiation energies, the resulting registration scenario is pseudo-multimodal and then the dissimilarity energy should be expressed in terms of a statistical measurement, such as the correlation ratio. However, the equalization performed in this work over the portal image tries to make the scenario monomodal and because of that a monomodal registration scheme has been used. In special cases, such as images with artifacts or deficient illumination a multimodal registration must be taken into account.

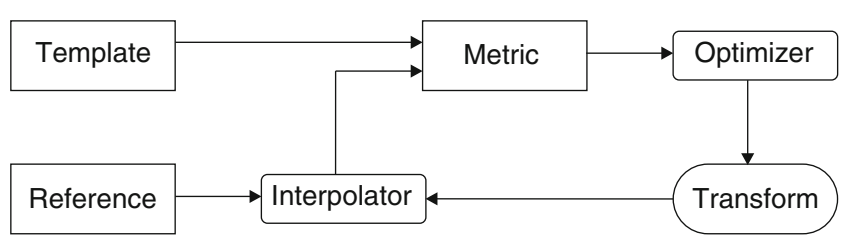

Fig. 6 Flow diagram of registration process
For the registration process, the ITK library [14] has been used. This library allows restricting the area to be registered, ignoring the rest of the image during the registration step. For our purposes, the region considered is the area delimited by the collimator box (see Fig. 2e) in the DRR image.

Given that the movements of the couch where the patient lies are restricted to purely translational displacements (longitudinal or transversal) only these transformations should be allowed. Therefore a rigid transformation without rotation could be chosen at the transformation step (see Fig. 6). Instead of directly using the algorithm provided by ITK, the frequency framework defined by the authors in ref. [15] is considered in this work, since it has proven its robustness and reliability on ill-conditioned medical image registration scenarios. The regularization term to be minimized is the diffusion term [16], which is based on the energy of first-order spatial derivatives of the displacement field, thus admitting constant displacements (i.e. rigid transformation) but penalizing oscillating deformations. The value of the parameter $\alpha$ has to be high enough in order to guarantee that estimating non-rigid transformations is unlikely. For all the experiments shown in this paper, $\alpha=$ $10^{4}$ works well.

ITK implements different interpolation methods, amongst which we have chosen linear interpolation because it gives better results than the nearest-neighbour method and is less complex than the spline interpolation. Thus, there is a trade-off between the simplicity of the computation and the quality of the interpolation, achieving similar results to the B spline.

The optimizer employed in this work is based on the frequency implementation of the steepest descent algorithm. As stated in [15], this strategy allows for an efficient implementation, which runs twice faster than most state-ofart approaches [17]. To avoid too high values of the step (learning parameter), every time the derivative direction changes abruptly, the optimizer assumes that a local maximum or minimum has been passed by and reacts halving the size of the step. After several decreases, the optimizer might be moving very slowly, hence a step of minimum size is defined. If the step reaches its minimum size, the algorithm has converged. If not, it will continue for a limited number of iterations.

Finally, and considering the scenario as monomodal, the metric employed is the sum of squared differences of the pixels from the portal and the DRR belonging to the region of interest. This metric is based on the fact that the intensity which represents equivalent points must be the same for both images. Under a multimodal scenario, the registration method remains the same except for the metric that would be the mutual information (MI). 
Fig. 7 Application example of the proposed methodology
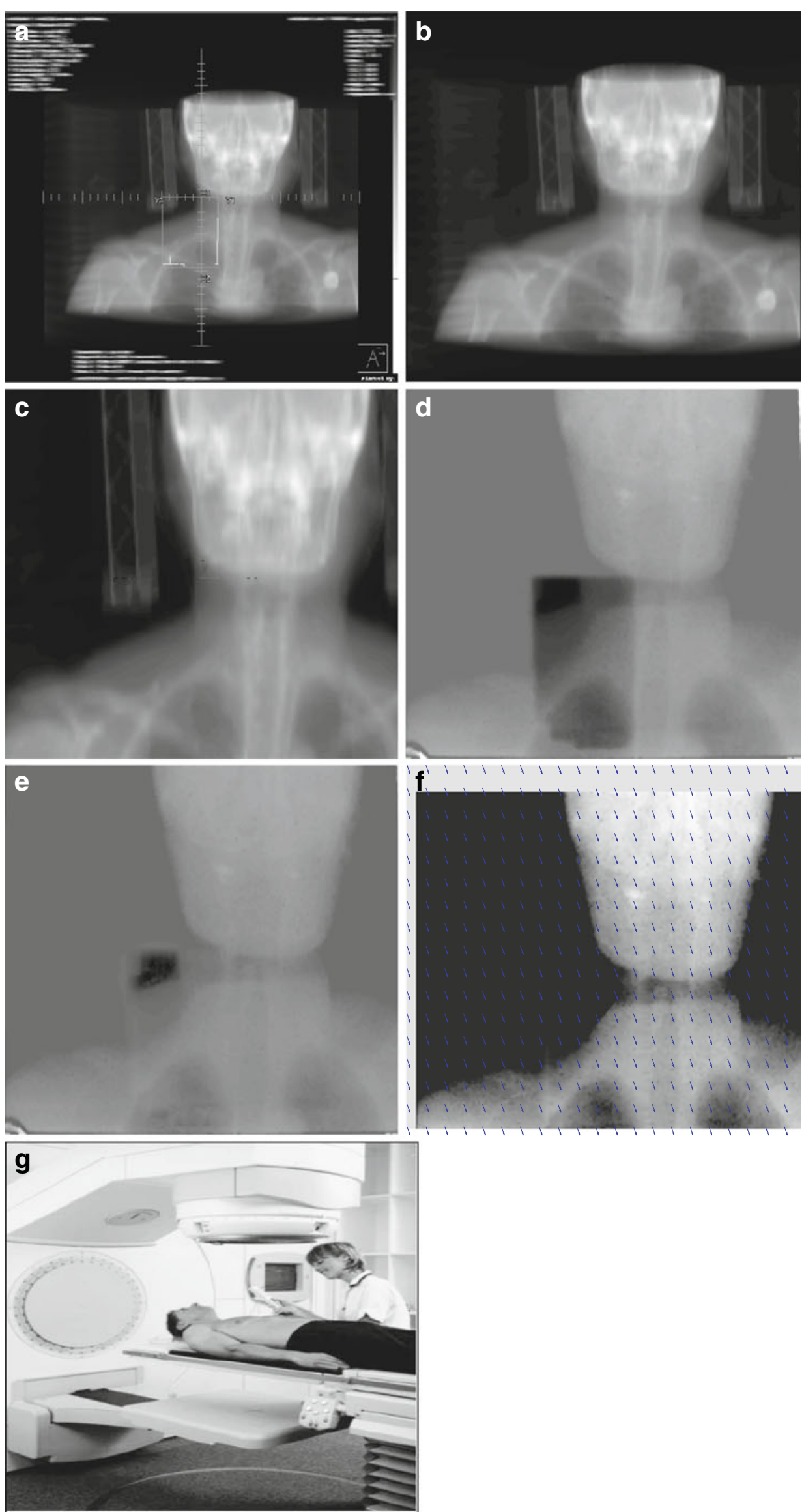
Table 1 Pairs of DRR-portal images after preprocessing, monomodal-registered images and displacements obtained in millimetres

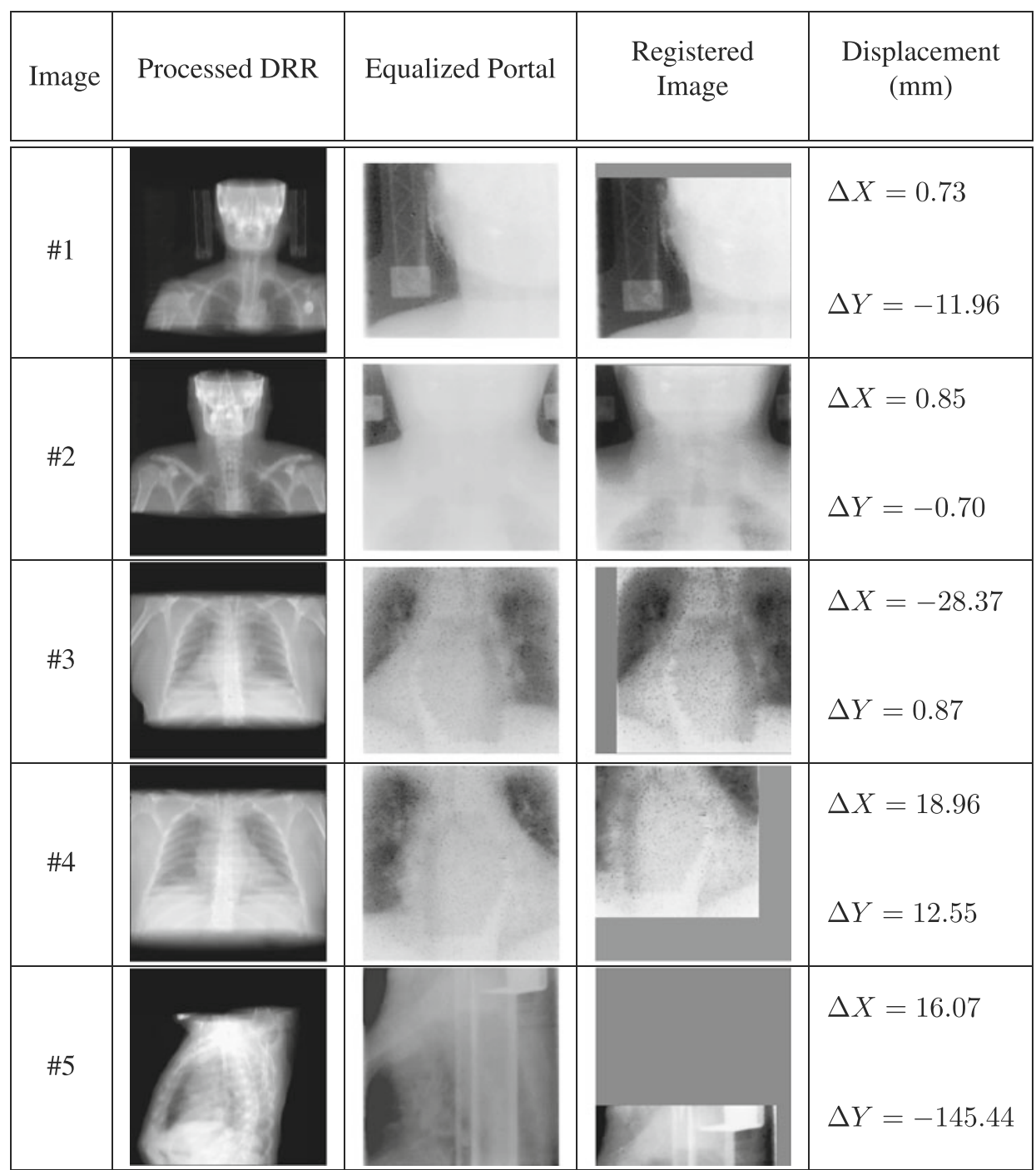

Experimental Results

In order to show the benefits of the proposed methodology over the scenario detailed in "Motivation and
Background", an actual clinical case will be considered in this subsection. Figure 7 gathers and illustrates the main steps of the method applied to a DRR-portal example pair. Figure $7 \mathrm{a}$ represents the original DRR, Fig. $7 \mathrm{~b}$ is the

Table 2 Pair of DRR-portal images, multimodal registered image and displacement obtained

\begin{tabular}{|c|c|c|c|c|}
\hline Image & Original DRR & Original Portal & $\begin{array}{l}\text { Registered } \\
\text { Image }\end{array}$ & $\begin{array}{l}\text { Displacement } \\
\text { (mm) }\end{array}$ \\
\hline \multirow{3}{*}{$\# 5$} & & & & $\Delta X=-5.12$ \\
\hline & & & & \\
\hline & & & & $\Delta Y=-8.22$ \\
\hline
\end{tabular}


cleaned DRR and Fig. 7d constitutes the original portal image (all these images are the same used as an example in previous sections). The processed DRR image (Fig. 7c) and the processed portal image (Fig. 7e) will be used as reference and template respectively in the registration problem context. Using the registration method described in section 2.3, the estimated displacement field $u(x)$, shown in Fig. 7f, is obtained. From that field, the correction of the position of the couch can be applied (see Fig. $7 \mathrm{~g}$ ). In the last iteration of the previously explained algorithm, the final and exact value of the couch displacement is extracted from the parameters of the transformation (transform block of the flow diagram in Fig. 6). In the present experiment, these computed couch movements are $11.96 \mathrm{~mm}$ downward and $0.7327 \mathrm{~mm}$ to the right. Nevertheless, the final assessment of that automatically calculated patient position concerns the radiologists at the hospital. This evaluation has been made at the result validation stage.

In Table 1, more registration examples are shown jointly with the computed displacement. For all the DRR-portal pairs, the displacement is accurately extracted, except for image 5. In this case, even though the accelerator print has been removed from the portal image (see Table 2), the intensity levels between both images will not match because of the artifacts introduced by the couch in the portal image. Thus, a metric based on MI is more suitable to reach a consistent registration. Consequently, a multimodal registration (MI metric) may be performed for this image pair. Both original images and final result after the new registration process are shown in Table 2. Note that now the portal image artifacts have no influence on the registration result and the couch displacement has been properly obtained.

\section{Discussion}

Although the results obtained can be considered as preliminary (its validity must be assessed by a doctor in a larger number of patients), the positionings of the couch provided by the automatic method present a good matching with those made by the radiologists during radiotherapy treatments. Therefore, the method gives the expected results.

It is remarkable that the non-shared information between reference (DRR) and template (portal) images, such as the axes or the auxiliary text in the first one or the accelerator print in the second one, could bring unexpected results in the image registration stage. For avoiding this inconvenience, some preprocessing of portal and DRR images is required as a previous step to the registration. Additionally, in our methodology these preprocessing steps are automat- ically achieved without needing human intervention, by means of morphological processing and other techniques of digital image processing that provide suitable images for the registration algorithm here proposed.

The effectiveness of considered metrics, sum of squared differences and mutual information, improves considerably after preprocessing, giving a major enhancement in the ultimate registration when compared with the registration without preprocessing. Consequently, the automatically computed displacements are more reliable, minimizing the variability introduced by visual inspection, whereas they are comparable to those established by the physician. Besides, the proposed rigid registration method implies a lightweight computational load and therefore is not time consuming.

Finally, note that proposed scheme allows certain flexibility if the involved images present some artifact. In such case, monomodal registration cannot be appropriate, since it could drive the algorithm to wrong displacements. Alternatively, multimodal registration can be used to overcome the artifacts, given that the used metric is not based on intensity likeness between images but on the mutual information between them.

Acknowledgements This work is funded by the Spanish "Ministerio de Educación y Ciencia" under the research project "Automatización de un sistema de planificación de radioterapia mediante técnicas emergentes de registro y fusion de imágenes biomédicas" (ref. TEC2006-13338/TCM). The authors would also like to thank the Radiology Department of the "Hospital Universitario Virgen de la Arrixaca" (Murcia, Spain) for providing all the images used in this paper and for their kind collaboration.

\section{References}

1. Wu J, Haycocks T, Alasti H, Ottewell G, Middlemiss N, Abdolell M, Warde P, Toi A, Catton C: Positioning errors and prostate motion during conformal prostate radiotherapy using on-line isocentre set-up verification and implanted prostate markers. Radiotherapy and Oncology 61(2):127-133, 2001

2. Yang Y, Xing L: Quantitative measurement of mlc leaf displacements using an electronic portal image device. Physics in Medicine and Biology 49(8):1521, 2004

3. Hashimoto S: Remote verification in radiotherapy using DRR and portal images: a pilot study. International Journal of Radiation Oncology, Biology, Physics 50:579-585, 2001

4. Modersitzki J: Numerical methods for image registration. Oxford University Press, New York, 2004

5. Matsopoulos GK, Asvestas PA, Delibasis KK, Kouloulias V, Uzunoglu N, Karaiskos P, Sandilos P: Registration of electronic portal images for patient set-up verification. Physics in Medicine and Biology 49(14):3279, 2004

6. Clippe S, Sarrut D, Malet C, Miguet S, Ginestet C, Carrie C: Patient setup error measurement using $3 \mathrm{~d}$ intensity-based image registration techniques. International Journal of Radiation Oncology*Biology*Physics 56(1):259-265, 2003

7. Kim YJ, Lee DH, Hong SH: A study on portal image for the automatic verification of radiation therapy. IEICE Trans Fundam 
Electron Commun Comput Sci (Inst Electron Inf Commun Eng) 82(6):945-951, 1999

8. Kunzler T, Grezdo J, Bogner J, Birkfellner W, Georg D: Registration of drrs and portal images for verification of stereotactic body radiotherapy: a feasibility study in lung cancer treatment. Physics in Medicine and Biology 52(8):2157, 2007

9. Khamene A, Bloch P, Wein W, Svatos M, Sauer F: Automatic registration of portal images and volumetric $\mathrm{CT}$ for patient positioning in radiation therapy. Medical Image Analysis 10:96-112, 2006

10. Serra J: Image analysis and mathematical morphology. Academic, London, 1982

11. Soille P: Morphological Image Analysis. Springer, Berlin, 1999

12. Bijhold J, Gilhuijs KGA, van Herk M, Meertens H: Radiation field edge detection in portal images. Physics in Medicine and Biology 36(12):1705, 1991

13. Chen, C.W., Lai, W., Yin, F.F., Chen, L.: Portal image feature extraction by hierarchical region processing technique. In: Systems, Man and Cybernetics, 1995. Intelligent Systems for the
21 st century. IEEE International Conference on. Volume 4. (2225) 3561-566 (1995)

14. Yoo TS, Ackerman MJ, Lorensen WE, Schroeder W, Chalana V, Aylward S, Metaxas D, Whitaker R: Engineering and algorithm design for an image processing api: A technical report on itk-the insight toolkit. Studies in Health Technology and Informatics 85:586-592, 2002

15. Larrey-Ruíz J, Verdú-Monedero R, Morales-Sánchez J: A Fourier domain framework for variational image registration. Journal of Mathematical Imaging and Vision 32:57-72, 2008

16. Fischer B, Modersitzki J: Fast diffusion registration. In: Nashed $\mathrm{MZ}$, Scherzer O Eds. Inverse problems, image analysis, and medical imaging. Contemporary Mathematics, vol. 313. AMS, Providence, 2002, pp 117-129

17. Verdú-Monedero R, Larrey-Ruíz J, Morales-Sánchez J: Frequency implementation of the Euler-Lagrange equations for variational image registration. IEEE Signal Processing Letters 15:321-324, 2008 